\title{
Caos y orden, complemento o dilema en las empresas, un análisis desde la complejidad
}

Chaos and Order, a Complement or a Dilemma in Companies, an Analysis Based on Complexity

Caos e ordem, complemento ou dilema nas empresas, uma análise desde a complexidade

\author{
Roberto Hidalgo-Flor \\ Universidad Estatal de Milagro. Milagro, Ecuador \\ robertohidalgo61@gmail.com \\ https://orcid.org/0000-0003-2369-3666
}

DOI: https://doi.org/10.32719/25506641.2022.11.3

Recibido: 15 de enero de 2021 - Revisado: 11 de febrero de 2021

Aceptado: 15 de abril de 2021 - Publicado: 1 de enero de 2022

Artículo de investigación

Licencia Creative Commons

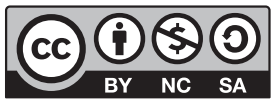




\section{Roberto Hidalgo-Flor}

\section{Resumen}

El artículo analiza, con base en los principios de comprensión de la complejidad que propone Edgar Morin, la relación de las empresas con elementos del entorno y de la sociedad de la que forman parte. Esto es necesario porque el enfoque tradicional, funcionalista, limita el análisis de lo que sucede y lo que pueden hacer las empresas para administrar su realidad y sus interrelaciones. Entonces, el objetivo de la investigación es estudiar y comprender el entorno que afecta a las empresas y sus influencias recíprocas, con base en el paradigma de complejidad. La metodología aplicada corresponde a una investigación netamente cualitativa, con entrevistas a profundidad a empresas y actores del entorno empresarial (consultores), aplicando análisis de contenido cualitativo. El estudio identifica cómo se presentan las ocho características o principios de complejidad en la relación entorno-empresas, la misma que se podría definir como una intermitencia de caos y orden. Sin embargo, la investigación refleja que, a la luz del paradigma de complejidad, ese caos aparente representa una opción de complementariedad, creadora y de crecimiento, en la que desorden, orden y organización constituyen un círculo virtuoso. Pero, además, que cada empresa, cada persona, cada situación, cada entorno es único y complejo, y de esa manera debe ser tratado.

Palabras clave: gestión, organización, empresa, administración, entorno.

JEL: M1Administración de empresas.

\section{Abstract}

Based on the principles for understanding complexity proposed by Edgar Morin, the article analyzes the relationship of companies with elements of the environment and the society that they are part of. This is necessary because the traditional, functionalist approach limits the analysis of what happens and what companies can do to manage their reality and their interrelationships. The purpose of the research is therefore to study and understand the environment that affects companies and their reciprocal influences, based on the paradigm of complexity. The applied methodology corresponds to a purely qualitative research, with in-depth interviews with companies and stakeholders in the business environment (consultants), using qualitative content analysis. The study identifies how the eight characteristics or principles of complexity arise in the environment-companies relationship, which could be defined as an intermittence of chaos and order. However, the research shows that, in light of the complexity paradigm, this apparent chaos represents an option of complementarity, creation, and growth, in which disarray, order, and organization constitute a virtuous circle; but also, that each company, each person, each situation, each environment is unique and complex, and must be treated accordingly.

Keywords: management, organization, company, administration, environment.

JEL: M1 Business Administration. 
Caos y orden, complemento o dilema en las empresas, un análisis desde la complejidad

\section{Resumo}

O artigo analisa, a partir dos princípios de compreensão da complexidade que propõe Edgar Morin, a relação das empresas com elementos do entorno e da sociedade da qual formam parte. Isto é necessário porque o enfoque tradicional, funcionalista, limita a análise do que acontece e do que podem fazer as empresas para administrar sua realidade e suas inter-relações. Então, o objetivo da pesquisa é estudar e compreender o entorno que afeta as empresas e suas influências recíprocas, desde o paradigma da complexidade. A metodologia aplicada corresponde a uma investigação nitidamente qualitativa, com entrevistas profundas a empresas e atores do entorno empresarial (consultores), aplicando a análise de conteúdo qualitativo. O estudo identifica como apresentam-se as oito características ou princípios da complexidade na relação entorno-empresas, a mesma que poderia definir-se como uma intermitência entre caos e ordem. Porém, a pesquisa reflete que, à luz do paradigma da complexidade, esse caos aparente representa uma opção de complementaridade, criadora e de crescimento, na que desordem, ordem e organização constituem um círculo virtuoso. Mas, ademais, que cada empresa, cada pessoa, cada situação, cada entorno é único e complexo e deve ser tratado como tal.

Palavras-chave: gestão, organização, empresa, administração, entorno.

JEL: M1 Administração de empresas.

\section{Introducción}

E 1 interés y necesidad de realizar esta investigación parte de la importancia de estudiar la dinámica de las relaciones organizacionales y sus vínculos con la sociedad; contribuye así a profundizar en una de las líneas de investigación de esta revista: la organización y gestión. Este trabajo considera que las empresas son sistemas complejos, desde el enfoque de Edgar Morin, que es una perspectiva distinta a la tradicional, el enfoque funcionalista, simplificador de realidades y generalizador de propuestas. Se usa una perspectiva amplia de la complejidad, que Morin (2006b) denomina como complejidad generalizada, en contraposición a la complejidad restringida, que es el enfoque de la ciencia clásica. En esta se ve lo complejo como sinónimo de lo complicado y se pretende modelizar y reproducir en laboratorio o en programas computacionales esas complicaciones, con el fin de establecer teorías o leyes de comportamiento y, a partir de ello, recetas de actuación. Esto ocurre tanto en las ciencias naturales como en las ciencias sociales y, entre ellas, en la gestión organizacional y empresarial. Un cambio de para- 
digma es la propuesta de Morin por lo que es necesario un análisis distinto, a partir de los principios de comprensión de la complejidad. Lo anterior determina diferentes prácticas y formas de gestionar las organizaciones, incluyendo la relación con la sociedad a la que pertenecen. El objetivo de esta investigación es estudiar y comprender el entorno que afecta a las empresas y sus influencias recíprocas, con base en el paradigma de complejidad, y lo hace para las medianas empresas de Quito.

\section{Marco teórico}

En este documento la reflexión sobre complejidad se sustenta en la propuesta de Edgar Morin, a quien se le reconoce entre los pensadores, filósofos y científicos sociales contemporáneos más prominentes (Solana 2005a; García 2006), cuyo trabajo es un esfuerzo "por construir una síntesis que permita pensar de modo integrado la complejidad organizacional del mundo físico, biológico y antroposocial desde una perspectiva anti-reduccionista, anti-esencialista, no finalista y no totalizadora" (Rodríguez-Zoya 2017, 2). Se considera que en términos filosóficos su enfoque es posmoderno (Woermann, Human y Preiser 2018) pues, adicionalmente a la identificación de características o relaciones causa-efecto en los sistemas complejos, induce a la reflexión y cuestionamiento de la observación del observador, tema que Niklas Luhmann lo había planteado desde el enfoque de la cibernética de segundo orden en los sistemas sociales (Ávila 2018) y que Morin lo profundiza con el proceso de conocimiento del observador.

Las experiencias de vida de Morin marcan su desarrollo intelectual, él promueve la transdisciplinariedad en la investigación. Las preguntas que orientan su obra son: ¿cuál es la estructura del conocimiento?, ¿cómo actúa el pensamiento? Morin considera que una estadía en California, teniendo 48 años, fue decisiva en su conocimiento y producción intelectual. Allí profundiza en teorías sobre sistemas, cibernética, comunicación, informática, dinámica de no equilibrio y caos, lo que contribuye a publicar, desde mediados de la década del 70, seis volúmenes de El Método (Vallejo-Gómez 2003; Solana 2005b). Por lo indicado, para comprender su obra conviene conocer las temáticas antes nombradas. 
Morin establece la necesidad de un nuevo paradigma para permitir cambios de pensamiento y de acción. Su preocupación es comprender la lógica del conocimiento, "no es solamente el conocimiento de la vida. Es al mismo tiempo el conocimiento del conocimiento de la vida" (Morin 1997, 28-29; cursiva del autor). Afirma que se requiere una reforma del entendimiento para comprender la complejidad (Morin 2001) y expresa que esta se ha entendido de una manera restringida o limitada, que es la manera en que lo han hecho los científicos modernos, pero la han enfocado desde lo que es complicado o difícil de explicar y la han abordado con modelización, formalización, identificación de leyes. Esto es lo que ha derivado en la ciencia de los sistemas complejos, sin que haya un replanteamiento epistemológico (Morin 2006b).

Esta complejidad restringida es distinta a lo que Morin llama complejidad generalizada, que parte del hecho de que todo sistema, y para él todo es un sistema (Morin 1994), puede considerarse complejo en su naturaleza de organización, pues no responde a una sola lógica o a una sola propiedad. Por esto, la complejidad generalizada es una cuestión epistemológica y de cambio de paradigma, requiere una nueva forma de pensar. Al plantearlo lo contrasta con el paradigma clásico y la necesidad de ruptura con él y de ampliar los horizontes del conocimiento, reconocer al desorden, a la oscuridad, a la ambigüedad, a la incertidumbre, a la contradicción, como fuentes de conocimiento (Morin 1998).

Ante la necesidad de método, Morin plantea estrategias para el conocimiento complejo (Solana 2005a), el cual "es una aventura en espiral [...] el descubrimiento de un principio simple no es el término; [lo] reenvía de nuevo al principio simple que ha esclarecido en parte" (Morin 1999a, 28). En esta perspectiva, el conocimiento es provisional y relativo en las ciencias naturales y en las ciencias sociales y humanas (Ruiz 2003). Las estrategias son los principios de intelección (entendimiento) de la complejidad que son factibles de aplicar a la comprensión de sistemas y fenómenos, abstractos y concretos. Él mismo lo hizo, principalmente para el pensamiento, pero, por ejemplo, también para la empresa (Morin 2011a) y varios autores lo han intentado también, con mayor o menor rigurosidad (Rodríguez-Zoya 2018).

Morin, en ocasiones, expone los principios de comprensión de la complejidad en forma contrapuesta a los de la ciencia clásica. Son esos principios 
los que sustentan las variables que se usan en esta investigación. En una de sus exposiciones, Morin (1999a) indica:

- La ciencia clásica (CC) propone la universalidad, generalizar el conocimiento; el paradigma de complejidad (PC) rescata y valora la singularidad, la unicidad de cada sistema, por lo que leyes que se suponen generalizables, son válidas solamente bajo determinadas circunstancias.

- La CC concibe un solo tiempo (el del progreso lineal, que también es el de entropía); el PC destaca la politemporalidad, en la que aparecen en forma atada: repetición, progreso, decadencia. Es la irreversibilidad, la evolución, la historicidad que planteaba Prigogine.

- La CC se fundamenta en la reducción (o elementalidad); entonces para conocer un sistema hay que descomponerlo en elementos. En el PC la elementalidad tiene límites, los elementos forman parte de conjuntos organizados y emergen cualidades que no existen en las partes. La interacción de los elementos es lo que da las características del todo.

- La CC se sustenta en las nociones orden-ley, las leyes deterministas reflejan la realidad. Morin plantea que lo que parece desorden es por la insuficiencia de conocimiento. Para el PC orden y ley son necesarios pero insuficientes, a mayor complejidad menos útil la idea de ley. Orden-desorden-organización tienen relación antagónica y complementaria, pero están en todo fenómeno organizado (Morin 1994).

- En la CC la causalidad es simple, es lineal, es exterior y superior a los objetos. En el PC el efecto no solo hace bucle con la causa (el efecto no solo retroactúa con la causa, que es lo que sustentaba la cibernética), sino que hay un juego complejo de endo (lo interno)-exo (lo externo)-causalidad, que se deben ver simultáneamente y no por separado (Morin 1999a).

- En la CC el fenómeno de organización es simple, las partes componen el todo y se acepta que el todo es más que la suma de las partes (así lo planteaba la teoría general de sistemas). En el PC, en la relación entre las partes y el todo, hay que considerar el principio de emergencia, relacionado a que las cualidades y propiedades que surgen (que emergen) en un todo, a partir de las interrelaciones entre las partes, retroactúan sobre ese todo. Hay que conocer el todo para conocer las partes y viceversa. En la sociedad, las interacciones entre individuos producen las características de esa sociedad, pero es esa sociedad la que produce a cada individuo. Morin 
lo denomina como principio hologramático: "la organización compleja del todo (holos) necesita la inscripción (engrama) del todo (holograma) en cada una de sus partes que, sin embargo, son singulares [...] al mismo tiempo son micro-todo virtuales" (Morin 2006, 113). En el principio de organización hay entonces un proceso de recursividad organizacional (de autoproducción).

- La CC parte de una disyunción entre objeto (sistema) y medioambiente, para entender al objeto, lo separa de su medioambiente, esto sustenta la experimentación; pero también plantea la disyunción completa entre objeto y sujeto que lo percibe y concibe. El PC plantea no separar sino distinguir al objeto de su medioambiente y considerar la relación entre el sujeto-observador-conceptuador y el objeto-observado-percibido-concebido. Por lo que es posible incluso considerar como sistema al observador y al sistema observado (el sistema sujeto-objeto), que también es susceptible de ser observado, constituyendo un nuevo sistema (en el caso del pensamiento, es el conocimiento del conocimiento).

- En la CC "las nociones de ser y de existencia estaban totalmente eliminadas por la formalización y la cuantificación [en el PC] han sido reintroducidas a partir de la idea de autoproducción" (Morin 1999a, 42). El ser y la existencia, que parecían categorías puramente metafísicas, están en el universo físico, pero al no ser una sustancia, el ser existe en la medida en que hay autoorganización. En el caso de individuos o grupos, pero también para las ideas y conocimientos, estos conceptos se pueden considerar en la perspectiva del principio de auto-eco-organización, en el que eco se refiere al ambiente, al entorno, que está constituido por la sociedad, los individuos, la cultura. Para esos sistemas (individuos, grupos, ideas, conocimientos) "allí donde haya a la vez auto-eco-organización y principio recursivo-hologramático, ya no hay determinismo mecánico, maquinería trivial, causalidad línea, reduccionismo brutal" (Morin 1998, 88).

- La CC se fundamenta en la lógica tradicional para establecer la verdad de las teorías. En el PC "todo sistema conceptual suficientemente rico incluye necesariamente cuestiones a las que no puede responder desde sí mismo, pero a las que solo puede responder refiriéndose al exterior de ese sistema" (Morin 1999a, 43); es decir, un metasistema, que a su vez requiere también otro metasistema, entonces el conocimiento no se pue- 
de clausurar. Lo anterior se relaciona también con el rol de la contradicción que, al ser insuperable en la lógica clásica, determina la necesidad de otra lógica (Morin 1999a).

De los principios anteriores, Morin (2011b) resume en tres la base para pensar y entender la complejidad: el principio dialógico, que implica que hay dos lógicas para abordar un tema, que pueden ser a la vez complementarias y antagonistas, este es el caso del orden y desorden que, en ocasiones, dan lugar a organización; el principio de recursividad organizacional "en el cual los productos y los efectos son, al mismo tiempo, causas y productores de aquello que los produce" (2011b, 106), y el principio hologramático que implica que el todo está en las partes y las partes están en el todo; pero también, lo que quizá presenta más "dificultades para nuestro entendimiento y nuestra estructura mental, el todo es más y, al mismo tiempo, menos que la suma de las partes" (2011a, 121-122; cursiva del autor).

Otros autores han establecido diversas propuestas para estudiar y caracterizar los sistemas complejos, de hecho, quienes contribuyeron a la evolución conceptual de la teoría de sistemas: Von Bertalanffy (teoría general de sistemas); Wiener y Ashby (cibernética de primer orden); Von Foerster, Luhmann, Bateson, Wallerstein (cibernética de segundo orden); Prigogine (sistemas dinámicos en no equilibrio); Rolando García (sistemas complejos) y otros autores que desarrollaron teorías relacionadas (información, comunicación, caos), consideraron que en los sistemas se presentaban características a las que denominaron con el adjetivo complejo; sin embargo, esas características de complejidad no siempre tenían el mismo enfoque y, en general, correspondían a lo que Morin denomina complejidad restringida.

Con base en lo anterior, el tratamiento de un fenómeno, desde la perspectiva del paradigma de complejidad, requiere ser tratado con un enfoque de comprenderlo y no solo describirlo o explicarlo. La comprensión, realizada de forma intelectual y objetiva, pero también de forma humana, es uno de los saberes que Morin (1999b) encuentra indispensables para construir una nueva civilización. El nuevo entendimiento, necesario para la comprensión de la complejidad, requiere una reforma tanto de la mente como de instituciones (por ejemplo, el sistema educativo) y esto, que puede parecer un círculo vicioso para la lógica clásica, solo se podrá hacer si poco a poco se desarrolla el pensamiento complejo (Morin 2006b). 


\section{Material y método}

Por la naturaleza cualitativa del estudio, se considera la propuesta que Creswell $(2007,37)$ plantea, en el sentido de comenzar "con suposiciones, una visión del mundo, el posible uso de una perspectiva teórica y el estudio de temas [...] mediante los que se indaga sobre el significado que, individuos o grupos, atribuyen a una situación social o humana". Lo anterior supone tomar decisiones teóricas y metodológicas con el fin de generar datos a partir de hechos sociales (Cohen y Gómez 2019), e implica combinar enfoques y estrategias de recolección de datos (Levitt et al. 2018), pero también priorizar variables a estudiar, el modo de obtenerlas y analizarlas, con el fin de cuidar una de las principales preocupaciones en las investigaciones cualitativas, esto es, el rigor y transparencia (Aguinis y Solarino 2019). Con esta referencia el diseño metodológico de la investigación se sustenta en: (i) teoría base: complejidad, que es la que mejor refleja a las organizaciones (Kornberger y Clegg 2003), con base en el enfoque de Morin; (ii) perspectiva epistemológica: subjetivismo, puesto que "dar cuenta de la realidad social exige conocer la actividad de los individuos, de los grupos y de las comunidades en cuanto interactividad [...] con un diálogo estructurado por la perspectiva de y desde el punto de vista del investigador [...] dejando en suspenso cuáles sean los significados auténticos, las experiencias reales y los hechos objetivos" (Bericat 1998, 75); adicionalmente, en un sistema complejo, por la superposición de sus atributos ontológicos y de las estrategias epistemológicas utilizadas para comprenderlos, es difícil que haya posiciones objetivas para estudiarlos (Woermann, Human y Preiser 2018); (iii) enfoque: fenomenología de tipo interpretativo (hermenéutica); (iv) método de construcción de conocimiento: inductivo, "parte de una realidad concreta [...] para llegar a una teorización posterior" (Albert 2007, 148); (iv) técnica de colecta de datos: entrevista a profundidad semiestructurada; (v) interpretación: análisis de contenido cualitativo, pues si bien el uso de herramientas computacionales se ha difundido y popularizado y, aunque "quizá sea excitante, [es algo] que no conduce a la comprensión" (Krippendorff 1997, 8) y más bien "estas son miradas reduccionistas que excluyen la posibilidad de representar propiedades de los objetos mediante categorías cualitativas" (Cohen y Gómez 2019, 24); (vi) tipo de investigación: descriptiva, con el fin de interpretar y comprender pro- 
cesos y comportamientos; (vii) unidad de investigación: medianas empresas de Quito (50 a 199 personas, ventas anuales entre USD 1000001 y USD 5000000 (EC 2011), no relacionadas o dependientes de empresas internacionales, para evitar influencias de matrices externas en su comportamiento), y también, complementariamente, consultores en temas de gestión y calidad, por ser actores del entorno de las empresas que conocen el ambiente y comportamiento empresarial.

Para organizar e interpretar los datos, Strauss y Corbin (2002) expresan que hay que elaborar categorías y codificar, lo que supone conceptualizarlos, reducirlos y relacionarlos y debe haber un procedimiento adecuado de codificación pues es parte de la validez y confiabilidad del estudio. Al revisar las categorías y códigos establecidos en la tabla siguiente, el lector podrá confirmar que corresponden a los principios de comprensión de la complejidad, explicados en el acápite anterior.

Tabla 1

Códigos y categorías para análisis de complejidad

\begin{tabular}{|c|l|l|}
\hline Variable & Código & \multicolumn{1}{c|}{ Categoría } \\
\hline \multirow{5}{*}{} & CE1 & $\begin{array}{l}\text { CE1 - Dialogicidad (presencia de dos lógicas antagónicas, por ejemplo, } \\
\text { orden-desorden). }\end{array}$ \\
\cline { 2 - 3 } & CE2 & $\begin{array}{l}\text { CE2 - Característica hologramática (el todo es mayor, igual, menor y/o } \\
\text { distinto que las partes). }\end{array}$ \\
\cline { 2 - 4 } & CE3 & $\begin{array}{l}\text { CE3 - Emergencia de cualidades (a partir del orden, del desorden o de las } \\
\text { interacciones internas o externas). }\end{array}$ \\
\cline { 2 - 3 } $\begin{array}{l}\text { Cacterísticas } \\
\text { de complejidad las empresas }\end{array}$ & CE4 & $\begin{array}{l}\text { CE4 - Multidimensionalidad (copresencia de hechos simples o complica- } \\
\text { dos: caóticos o no; deterministas o probabilísticos; evolutivos). }\end{array}$ \\
\cline { 2 - 3 } & CE5 & $\begin{array}{l}\text { CE5 - Politemporalidad (decadencia, repetición, progreso, entropía nega- } \\
\text { tiva (neguentropía). }\end{array}$ \\
\cline { 2 - 3 } & CE6 & $\begin{array}{l}\text { CE6 - Recursividad organizacional (autoproducción, autoorganización, } \\
\text { endo-exo-causalidad; flexibilidad, adaptabilidad). }\end{array}$ \\
\cline { 2 - 3 } & CE7 & $\begin{array}{l}\text { CE7 - Relación sistema-entorno (relación objeto-medioambiente; no dis- } \\
\text { yunción, uno influye en otro y viceversa). }\end{array}$ \\
\cline { 2 - 3 } & CE8 & $\begin{array}{l}\text { CE8 - En la empresa: estrategia (lo emergente) frente a programación } \\
\text { (lo planificado). }\end{array}$ \\
\hline
\end{tabular}

Elaboración propia. 
La selección de la muestra y su adecuada justificación es una de las falencias en las investigaciones cualitativas (Ashworth, McDermott y Currie 2019), lo que se ha querido evitar en este caso. Una de las opciones es "hacer por casos extremos, típicos o por la máxima varianza” (Albert 2007, 178), pero también se hace por conveniencia, casos consecutivos o cuotas. Como parte del muestreo por conveniencia está la muestra por criterio, en la que "además de encontrarse disponibles los sujetos de estudio, se elige a los que se presuponen más apropiados" (Reyes, Espinoza y Olvera 2013, 2922); autores como Creswell y Creswell (2018) usan la palabra intencional; Hernández-Sampieri, Fernández y Baptista (2010) indican que es por propósito. Para esta investigación se seleccionó por criterio a un grupo de empresas y de consultores (con lo que se cumple también con la característica de convergencia o triangulación, deseable para este tipo de metodología).

En relación con el número de entrevistas, se consideró que: (i) "es posible dar inicio a una investigación de tipo cualitativo con una muestra de aproximadamente 12 participantes" (Reyes, Espinosa y Olvera 2013, 2922); ${ }^{1}$ (ii) el criterio de saturación es el que se utiliza para limitar el número de entrevistas puesto que el investigador, al utilizar un diseño emergente, de acuerdo con las circunstancias, y no completamente predefinido previamente "no será capaz de especificar el tamaño de la muestra [...] para comprender totalmente un fenómeno de interés" (Albert 2007, 179), lo que significa que la saturación se da cuando "no surgen registros nuevos [...], en el juego entre las variables y la realidad de estudio no se producen nuevos datos" (Cohen y Gómez 2019, 252). Hennink, Kaiser y Marconi (2017) refieren la dificultad de encontrar en investigaciones cualitativas los criterios utilizados para establecer que se ha alcanzado la saturación; exponen dos estudios de otros autores y uno propio y concluyen que "la saturación de código [o de temas] se alcanzó en 9 entrevistas, en las que se identificó el rango de asuntos temáticos. Sin embargo, se necesitaron de 16 a 24 entrevistas para alcanzar la saturación de significado" (591). A partir de esto recomiendan parámetros de referencia para establecer la saturación y con ello una determinación de la muestra. Estos parámetros, explicados por Serna (2019), determinan que

1. Estudios locales han aplicado una muestra similar, ejemplo, Oviedo y López (2020) tiene 13. 
se compare entre posiciones extremas para identificar lo que requiere el tema estudiado, específicamente:

- Propósito del estudio: caracterización de temas específicos versus desarrollo de teoría.

- Características de la población: homogénea versus heterogénea.

- Estrategia de muestreo: con criterios flexibles versus estrictos.

- Calidad de los datos: básicos, pero suficientes para una saturación de códigos, versus profundos, necesarios para desarrollar teoría (en inglés los autores usan thin y contraponen con thick, en este documento se ha preferido utilizar básicos y profundos).

- Tipos de variables (y de códigos): orientados a temas concretos versus desarrollar conceptos.

- Base de códigos: relativamente predefinidos, estables, versus emergentes en el proceso de investigación.

- La meta de saturación: en códigos (definida como el punto en el que no se identifican aportes adicionales y el libro de códigos — variables - comienza a estabilizarse) versus en significados (definida como el punto en el que no se pueden encontrar más dimensiones, matices o percepciones de los problemas), lo que queda a criterio del investigador.

Para el estudio que corresponde a este artículo hay que considerar que: la base de variables está prácticamente predefinida por el marco teórico y son puntos concretos para caracterizar un tema; no tiene una perspectiva de desarrollo de teoría sino de comprensión de un fenómeno; la estrategia de muestreo es flexible, sustentada en opinión de expertos (los consultores). Por lo anterior, las características se acercan más a tener una saturación por códigos que, de acuerdo a Hennink, Kaiser y Marconi (2017), significa que es posible una muestra con 9 entrevistas, aunque por un parámetro (heterogeneidad de la población considerada), que es solo 1 entre 7, la muestra podría estar entre 10 y 12. De acuerdo con lo indicado, el número de entrevistas planificadas fue 12. En la práctica se ejecutaron 13, se validaron 11 y se consideró haber alcanzado la saturación. 


\section{Resultados}

El objetivo de la investigación fue estudiar y comprender el entorno que afecta a las empresas y sus influencias recíprocas, con base en el paradigma de complejidad, contrario al análisis tradicional funcionalista. En este acápite se presentan, como ejemplo, algunas frases de los entrevistados que tienen que ver con la relación empresa-entorno; es decir, la relación objeto-medio ambiente que, desde la complejidad, se debe ver en una perspectiva de no disyunción puesto que uno influye en otro y viceversa. Cabe aclarar que en las entrevistas se profundizó en la evolución de las empresas desde su creación, por lo que algunos de los temas expuestos corresponden a situaciones ocurridas hace algunos años. A continuación, se transcriben las frases y luego, entre paréntesis, se indica la interpretación hecha por el investigador, que obviamente es subjetiva, tal como se estableció y justificó en el acápite de material y método, y que puede ser cuestionada por el lector desde su propia perspectiva. Posteriormente se analizan los resultados, en función de cada categoría utilizada.

\footnotetext{
Empresa 1: - "Pero se contrarrestó con producción local propia" (respuesta de la empresa al entorno).

- "A veces no se sabe qué autoridad rige" (marco institucional).

- "Los asesores nos capacitaron, nos orientaron, ellos tienen una plataforma" (influencia de consultores, inciden en que se repliquen procesos en las empresas).

Empresa 2: - "Hay bastante afectación de las normas y regulaciones. Para mí, hay mucho impuesto [...] en cuanto a normas, reglas que se deban seguir son muy buenas, yo estoy muy de acuerdo en que haya entes de control que vengan y nos digan lo que tenemos que tener" (dos lógicas -las regulaciones: malas, pero también buenas, dependiendo de lo que interesa).

- "Hubo la erupción del volcán Pichincha y eso fue una cosa terrible [...] y peor para el sector hotelero" (influencia de la naturaleza).

- "Yo no me fijo mucho en la competencia, no hay un competidor principal al que quiera emular [...] Hacemos estudios de mercado, claro que casi mensualmente estamos chequeando tarifas, viendo qué hace la competencia" (dos lógicas -lo que se dice y lo que se hace ante la competencia).

- "Hemos dado comentarios a reglamentos que se están elaborando por parte de

Quito Turismo" (influencia de la empresa en el entorno).
} 


\begin{abstract}
Empresa 3: - "El gobierno decidió imponer aranceles que afectaron a la fabricación nacional de partes y piezas para línea blanca" (influencia de regulaciones).

- "La influencia de los clientes en el rumbo de la empresa es definitiva" (influencia clientes).

Empresa 4: $\quad$-Se intentó el negocio de la comercialización de energía y aplicaciones de telemetría. Lamentablemente el país [...] no ha desarrollado hacia eso" (influencia de falta de regulaciones).

Empresa 5: - "El cambio de normativa en el Pacto Andino afectó" (influencia de regulaciones internacionales).

- "La situación económica local e internacional sí ha afectado. En los años 90 la crisis de los Estados Unidos, después la crisis internacional, bajaron nuestras ventas, y los últimos años la crisis en el país" (efectos multidimensionales).

Empresa 6: "Una de las empresas de las que se tiene representación cedió a otra empresa esa representación" (influencia de proveedores).

- "Después de dos años se restableció por pedido de los propios clientes" (influencia positiva de clientes).

Consultor 1: - "Si el cliente es el Estado, les va a pedir una certificación ISO" (influencia de clientes).

Consultor 3: - "Necesidad de sistemas informáticos [...] por la presión que el Servicio de Rentas Internas (SRI) ha estado ejerciendo" (influencia de regulaciones e instituciones).

- "Resaltamos que [...] empresas importantes ya tienen algo y ellos no [...] nosotros no tratamos de vender algo que no le sirva a la empresa, en eso hay que tener cuidado, pero posiblemente hay gente que sí lo hace" (influencia de consultores).

Consultor 5: - "Lo más determinante para que las empresas adopten el uso de instrumentos de gestión empresarial ha sido la competencia y el mercado" (influencias de competidores, de clientes y de proveedores).
\end{abstract}

Elaboración propia.

\title{
Discusión y análisis de resultados
}

La información recopilada podría interpretarse como una intermitencia de caos y orden en la relación empresa-entorno, si el análisis fuera solamente funcionalista, pero - a la luz del paradigma de complejidad - se puede ver de una manera distinta, que es lo que se pretende en este artículo. En el análisis con los principios de complejidad generalizada, en la relación empresa-entorno (categoría CE7), se ve que el sistema empresa es parte de un sistema entorno que lo engloba, lo condiciona, lo determina, pero al cual 
también la empresa influye y afecta, y que ambos pueden conceptuarse como otro sistema. En este entorno está el rol de los diversos agentes que lo componen, lo cual se ha identificado en las frases recopiladas en la investigación (que son muchas más que las presentadas anteriormente como ejemplo), pero están también visibles los efectos positivos o negativos que pueden generarse, lo que se corrobora con hallazgos en otras investigaciones (Benzaquen y Pérez 2016; Simon y Kafel 2018). Se aprecia la influencia, en diversos ámbitos, de entidades reguladoras (el SRI), de los clientes, de los proveedores, de los consultores, incluso se ve el efecto de la naturaleza, los temas políticos, la situación económica.

Lo anterior determina la necesidad de estrategias emergentes en las empresas (categoría CE8), en contraposición a lo que se pudo haber planificado, ante los cambios del entorno que los empresarios interpretan a veces como situaciones caóticas. En la concepción de Morin (2011a) la estrategia (lo emergente) supera a la planificación, que él la identifica más como una programación específica y estática; el funcionalismo privilegia la planificación. En las frases presentadas se refleja también que emergerán cualidades (categoría CE3), relacionadas a hacer nuevas cosas o cambiar lo que se hace (en productos, servicios o procesos), también modificar la estructura organizacional. Se evidencia que, ante situaciones aparentemente caóticas, es decir el desorden, se llega al orden y a la organización (reestructuración o reordenamiento de la empresa), es decir, la auto-eco-organización, constatándose la recursividad organizacional (categoría CE6), tema que el funcionalismo no considera.

La presencia de lógicas distintas (categoría CE1) pero coexistentes en el diario desempeño de las empresas se hace evidente cuando, en las frases recopiladas, se contraponen lo ideal y lo pragmático; el deber ser y la resistencia al deber ser; la planificación y la necesidad de acciones emergentes; la valoración pero también resistencia a las regulaciones y a la normatividad; el reconocimiento y la negación de que la competencia es objeto de interés, observación y quizá copia (Wongthongchai y Saenchaiyathon 2019). En el análisis funcionalista lo anterior no se considera.

En la relación empresa-entorno están presentes, simultáneamente y generando efectos positivos y/o negativos, diversidad de hechos o situaciones (categoría CE4). Algunas frases indican que hay incidencia de varios actores 
y las evaluaciones que se hagan deben tener presente también varias dimensiones (lo indica el consultor 5). En el funcionalismo, al separar y simplificar los elementos bajo análisis, el efecto de la simultaneidad se diluye.

En el principio de politemporalidad (categoría CE5, no considerada por el funcionalismo) se ve que el avance del tiempo no es lineal, que incluso puede retroceder y que puede significar avances y retrocesos; fracasos y adelantos. Politemporalidad en la que problemas y soluciones no solo coexisten, sino que se dinamizan entre ellos, los problemas que crean oportunidades (empresa 1). Aparentes retrocesos pueden significar avances.

La característica hologramática (categoría CE2) no se evidenció directamente en las entrevistas, pero definitivamente está presente al observar el funcionamiento empresarial, en su estructura y en la cultura organizacional. Esto es así porque, al mismo tiempo, la empresa hace más de lo que hace una de sus áreas (por ejemplo, la empresa no es solo el área de producción), pero también hace menos de lo que pudiera hacer una de ellas (si hay sobrecapacidad en el área de producción). Lo que la empresa hace es distinto, en conjunto, de lo que hace cada área. Pero también, ciertas características del todo, por ejemplo, la cultura organizacional, está en las partes, que a su vez contribuyen a crear esa cultura organizacional. Se establece así que el todo es más, pero también menos que sus partes; es distinto, pero las partes están en el todo y el todo está en las partes. Una reflexión como la anterior no es parte del análisis funcionalista, que posiblemente se limitará a establecer la productividad en cada área y plantear requerimientos de coordinación.

\section{Conclusiones}

En esta investigación se ha caracterizado la complejidad que se presenta en la relación empresas-entorno que, en una primera instancia, se puede ver como una relación en la que el orden y el caos están presentes, pero sin una aparente implicación entre ellos. El análisis de complejidad, a través de los principios planteados por Edgar Morin, permite identificar que, más allá de ese aparente desencuentro, hay una opción de complementación y de comprender y proyectar lo que sucede. 
Esta posibilidad de aprender de y aprehender un fenómeno desde la complejidad, implica no solo aplicar para el análisis, sino aceptar y comprender en el día a día los principios planteados por Morin, principalmente los siguientes: el de singularidad (unicidad de cada sistema); el de dialogicidad (lógicas antagónicas o complementarias que coexisten); el principio hologramático (la relación entre el todo y las partes); el principio de recursividad organizacional (dada una causa y su efecto, el efecto puede ser causa de su causa; se presentan endo-exo-causalidad y autoproducción); la auto-eco-organización relacionada al principio de emergencia (de cualidades). En todo sistema complejo estos principios se presentan en forma conjunta, integral.

A partir del ejercicio realizado se establece que fenómenos identificados e inicialmente caracterizados por marcos teóricos de enfoque funcionalista, modernista, se pueden estudiar en la perspectiva del paradigma de complejidad (cuyo enfoque es posmodernista), lo que abre opciones de comprenderlos en nuevas dimensiones y proyectarlos para un mejor desempeño organizacional.

La realidad encontrada en las empresas muestra que la complejidad está allí, en esos sistemas: en las empresas y los fenómenos que se presentan en ellas. Comprender esa complejidad es el reto. El nuevo entendimiento que se requiere, parafraseando a Morin (2006b), necesita una reforma tanto de la mente como de las instituciones (por ejemplo, el sistema educativo) y esto solo se podrá hacer si poco a poco se desarrolla el pensamiento complejo. En esta medida, el paradigma de complejidad representa una opción creadora y de crecimiento, en la que desorden, orden y organización constituyen un círculo virtuoso. Un cambio de paradigma es la propuesta de Morin y, en esa línea, es necesario un análisis distinto y diferentes prácticas y formas de gestionar las organizaciones, incluyendo su relación con el entorno, la sociedad a la que pertenecen.

Esta investigación contribuye a identificar y reflexionar sobre la riqueza de la individualidad de cada empresa, de quienes la conforman y de la relación con el entorno. El funcionalismo puede pretender uniformidad que garantice determinados resultados, pero lo real es la diversidad; el reto es aprovechar esa diversidad de la mejor manera, enriquecerla y permitir su desarrollo. Instituciones, programas o proyectos que pretendan promover mejoras en la gestión empresarial deberán evitar que se quiera uniformizar, porque no lo van a lograr. El estudio realizado refleja que cada empresa, cada 
persona, cada situación, cada entorno es único y complejo, y de esa manera debe ser tratado.

\section{Referencias}

Aguinis, Herman, y Angelo Solarino. 2019. "Transparency and Replicability in Qualitative Research: The Case of Interviews with Elite Informants". Strategic Management Journal 40: 1291-1315. https://doi.org/10.1002/smj.3015.

Albert, María José. 2007. La investigación educativa: claves teóricas. Madrid: McGraw-Hill.

Ashworth, Rachel, Aoife McDermott y Graeme Currie. 2019. "Theorizing from Qualitative Research in Public Administration". Journal of Public Administration Research and Theory 29 (2): 318-333. https://doi.org/10.1093/jopart/muy057.

Ávila, Juan Pablo. 2018. "Ilustración sociológica: de la crítica a la observación de segundo orden en la sociología de Niklas Luhmann". Economía y Política 5 (2): 67-86. http://bit. ly/3aLiIWu.

Benzaquen, Jorge, y Maximiliano Pérez. 2016. "El ISO 9001 y TQM en las empresas de Ecuador". Journal Globalization, Competitiveness and Governability - GCG 10 (3): 153176. https://doi.org/10.3232/GCG.2016.V10.N3.06.

Bericat, Eduardo. 1998. La integración de los métodos cuantitativo y cualitativo en la investigación social. Barcelona: Ariel.

Cohen, Nestor, y Gabriela Gómez. 2019. Metodología de la investigación, ¿para qué?: la producción de los datos y los diseños. Buenos Aires: Teseo / CLACSO.

Creswell, John. 2007. Qualitative Inquiry and Research Design. Thousand Oaks: Sage Publications.

Creswell, John, y David Creswell. 2018. Research Design: Qualitative, Quantitative and Mixed Methods Approaches. Thousand Oaks: Sage Publications.

EC. 2011. Decreto Ejecutivo 757. Registro Oficial 450, Suplemento, 17 de mayo.

García, Rolando. 2006. Sistemas complejos. Barcelona: Gedisa.

Hennink, Monique, Bonnie Kaiser y Vincent Marconi. 2017. "Code Saturation Versus Meaning Saturation: How Many Interviews are Enough?”. Qualitative Health Research 27 (4): 591-608. https://doi.org/10.1177/1049732316665344.

Hernández-Sampieri, Roberto, Carlos Fernández y María Baptista. 2010. Metodología de la investigación. Ciudad de México: McGrawHill.

Kornberger, Martin, y Stewart Clegg. 2003. "The Architecture of Complexity". Culture and Organization 9 (2): 75-91. http://dx.doi.org/10.1080/14759550302804.

Krippendorff, Klaus. 1997. Metodología de análisis de contenido. Barcelona: Paidós. 
Caos y orden, complemento o dilema en las empresas, un análisis desde la complejidad

Levitt, Heidy, Michael Bamberg, John Creswell, David Frost, Ruthellen y Carola Suárez-Orozco. 2018. "Journal Article Reporting Standards for Qualitative Primary, Qualitative Meta-Analytic, and Mixed Methods Research in Psychology". American Psychologist 73 (1): 26-46. http://dx.doi.org/10.1037/amp0000151.

Morin, Edgar. 1994. "Epistemología de la complejidad". En Nuevos paradigmas, cultura y subjetividad, coordinado por Dora Fried, 421-442. Buenos Aires: Paidós.

--- 1997. El Método II. La vida de la vida. Madrid: Ediciones Cátedra.

--- 1998. El Método IV. Las ideas. Madrid: Ediciones Cátedra.

--- 1999a. "La epistemología de la complejidad". En Con Edgar Morin, por un pensamiento complejo: implicaciones interdisciplinarias, coordinado por José Luis Solana, 27-52. Madrid: Universidad Internacional de Andalucía.

---. 1999b. Los siete saberes necesarios para la educación del futuro. Bogotá: UNESCO.

---. 2001. El Método I. La naturaleza de la naturaleza. Madrid: Ediciones Cátedra.

---. 2006a. El Método III. El conocimiento del conocimiento. Madrid: Ediciones Cátedra.

---. 2006b. "La complejidad hoy". Video de YouTube, a partir de una ponencia presentada en el CEIICH de la Universidad Autónoma de México. Accedido diciembre de 2020. https:// bit.ly/3rxA7IW.

---. 2011a. "La complejidad y la empresa". En Introducción al pensamiento complejo, editado por Marcelo Pakman, 121-131. Barcelona: Gedisa.

---. 2011b. "El paradigma de complejidad". En Introducción al pensamiento complejo, 87110. Barcelona: Gedisa.

Oviedo, Mariela, y Marcelo López. 2020. "Una aproximación a lo cualitativo: identificando las creencias de la compartición de conocimiento". Estudios de la Gestión 7: 19-42. https://doi.org/10.32719/25506641.2020.7.1.

Reyes, Octavio, Rafael Espinoza y Ramón Olvera. 2013. "Criterios para determinar el tamaño de muestra en estudios descriptivos". Congreso Internacional de Investigación de Celaya 5 (3): 2919-2924. http://bit.ly/3abP76U.

Rodríguez-Zoya, Leonardo. 2017. “Contribución a la crítica del pensamiento de Edgar Morin. Bases para un programa de investigación sobre los paradigmas". Gaceta de Antropología 33 (2):1-15. http://bit.ly/3pZETPe.

--- 2018. La emergencia de los enfoques de la complejidad en América Latina. Buenos Aires: Comunidad Editora Latinoamericana.

Ruiz, Luis Enrique. 2003. "La complejidad en las ciencias sociales". En Manual de iniciación pedagógica al pensamiento complejo, compilado por Marco Antonio Velilla, 249-256. Quito: UNESCO.

Serna, Miguel. 2019. "¿Cómo mejorar el muestreo en estudios de porte medio usando diseños con métodos mixtos? Aportes desde el campo de estudios de élites". Empiria, Revista de Metodología de Ciencias Sociales 43: 187-210. https://doi.org/10.5944/empiria.43.2019.24305. 
Simon, Alexandra, y Piotr Kafel. 2018. "Reasons for Decertification of ISO 9001. An Empirical Study". Innovar: Revista de Ciencias Administrativas y Sociales 28 (70): 69-80. http:// dx.doi.org/10.15446/innovar.v28n70.74449.

Solana, José Luis. 2005a. "Prólogo". En Con Edgar Morin, por un pensamiento complejo: implicaciones interdisciplinarias, editado por José Luis Solana, 9-25. Madrid: Ediciones Akal / Universidad Internacional de Andalucía.

---. 2005b. “Anexo: Un recorrido por la vida y la obra de Edgar Morin”. En Con Edgar Morin, por un pensamiento complejo: implicaciones interdisciplinarias, editado por José Luis Solana, 225-236. Madrid: Ediciones Akal / Universidad Internacional de Andalucía.

Strauss, Anselm, y Juliet Corbin. 2002. Bases de la investigación cualitativa. Técnicas y procedimientos para desarrollar la teoría fundamentada. Medellín: Universidad de Antioquia.

Vallejo-Gómez, Nelson. 2003. "Morin, pensador de la complejidad". En Manual de iniciación pedagógica al pensamiento complejo, compilado por Marco Antonio Velilla, 129-145. Quito: UNESCO.

Woermann, Minka, Oliver Human y Rika Preiser. 2018. "General Complexity: A Philosophical and Critical Perspective". Emergence: Complexity and Organization 20 (2): 1-16. https://bit.ly/2YU2BQT.

Wongthongchai, Jirawat, y Krittapha Saenchaiyathon. 2019. "The Key Role of Institution Pressure on Green Supply Chain Practice and the Firm's Performance”. Journal of Industrial Engineering and Management 12 (3): 432-446. https://doi.org/10.3926/jiem.2994. 